\title{
Bilateral Vocal Cord Dysfunction in the Setting of Lateral Medullary Infarction: A Case Report and Review of Neuro-Anatomical Correlate
}

\section{Babi MA ${ }^{1,2^{*}}$, Waheed $\mathbf{W}^{1}$, Aljerdi $\mathbf{S}^{1}$, Vizzard $\mathbf{M A}^{1}$ and Gorman $\mathbf{M J}^{1}$}

${ }^{1}$ Department of Neurological Sciences, The University of Vermont Medical Center and the University of Vermont College of Medicine. 111 Colchester Avenue, Burlington VT 05401.

${ }^{2}$ Division of neuro-critical care, Department of Neurology. Duke, University Hospital. 2301 Erwin Rd, Durham NC 27710, USA

${ }^{*}$ Corresponding author: M Ali Babi, 2Division of neuro-critical care, Department of Neurology, Duke University Hospital. 2301 Erwin Rd, Durham NC 27710, USA. Tel: 802-777-2880; Fax: 802-847-5690; E-mail: ncc.babi@gmail.com

Received date: September 21, 2015; Accepted date: December 02, 2015; Published date: December 09, 2015

Copyright: $\odot 2015$ Babi MA, et al. This is an open-access article distributed under the terms of the Creative Commons Attribution License, which permits unrestricted use, distribution, and reproduction in any medium, provided the original author and source are credited.

\begin{abstract}
Background: Lateral medullary (Wallenberg) syndrome is the most common stroke syndrome related to vertebral artery disease. The hallmark of this syndrome is crossed sensory findings affecting the contralateral trunk and extremities and the ipsilateral side of the face. This is in addition to dysarthria, dysphagia, vertigo, and Horner's syndrome. Unilateral vocal cord dysfunction is frequently seen, but bilateral vocal cord paralysis is an extremely rare and potentially life threatening complication.

Case Presentation: We present the case of a 75 year-old man who presented with the diagnosis of acute lateral medullary (Wallenberg) syndrome, and experienced bilateral vocal cord paresis leading to acute airway obstruction. Conclusion: We report a rare manifestation of acute ischemic stroke: bilateral vocal cord paresis. We propose an underlying neuroanatomical mechanism for bilateral vocal cord involvement.
\end{abstract}

Keywords: Stroke; Vocal cord paralysis; Brainstem stroke; Ischemic stroke; Lateral medullary syndrome; Autonomic dysfunction; Airway obstruction; Respiratory failure

\section{Introduction}

Lateral medullary syndrome, also known as Wallenberg syndrome, is the most common stroke syndrome related to vertebral artery disease. This clinical syndrome was described over two centuries ago by Gaspard Vieusseux of Geneva, at the Medical and Chirurgical Society of London;

"Vertigo, unilateral facial numbness, loss of pain and temperature appreciation in the opposite limbs, dysphasia[sic] and hoarseness, minor tongue involvement, hiccups (cured by taking up the habit of a morning cigarette) and a drooped eyelid" [1]. However, it was not until 1895 that accurate localization through autopsy was established by Adolf Wallenberg [2].

Lateral medullary infarction arises either due to occlusive disease affecting the posterior inferior cerebellar artery (PICA), or due to occlusion of small feeder branches arising directly from the vertebral artery itself $[3,4]$. It is characterized by specific features (Figure $1(\mathrm{a}, \mathrm{b})$ ) that make it distinct from other posterior circulation syndromes, albeit often missed by non-neurologists [3].

Many complications of lateral medullary ischemia have been reported. These include cardiac arrhythmias, respiratory dysfunction, and dysautonomia. The latter may manifest as bradycardia, sinus pauses, sinus tachycardia, ventricular tachycardia / fibrillation, premature ventricular complexes (PVCs), orthostasis without cardiac rate response, atrial fibrillation, and tachyarrythmias. These complications can lead to sudden cardiac death, even during the convalescence phase after stroke [5-8].
Bilateral vocal cord paresis (VCP), resulting in airway obstruction, is a very rare complication of lateral medullary syndrome. VCP has best been described in the setting of neck surgery, neck malignancies, and endotracheal intubation, in addition to idiopathic cases $[4,9,10]$. VCP has also been described with an array of neurological syndromes, including Arnold-Chiari malformation, meningomyelocele, amyotrophic lateral sclerosis (ALS), myasthenia gravis, Mobius syndrome, Charcot-marie-tooth syndrome, post-polio syndrome, ShyDrager syndrome, Creutzfeldt-Jacob syndrome, neurolyme, neurosarcoidosis, neurosyphilis, and collagen-vascular disease $[9,10]$

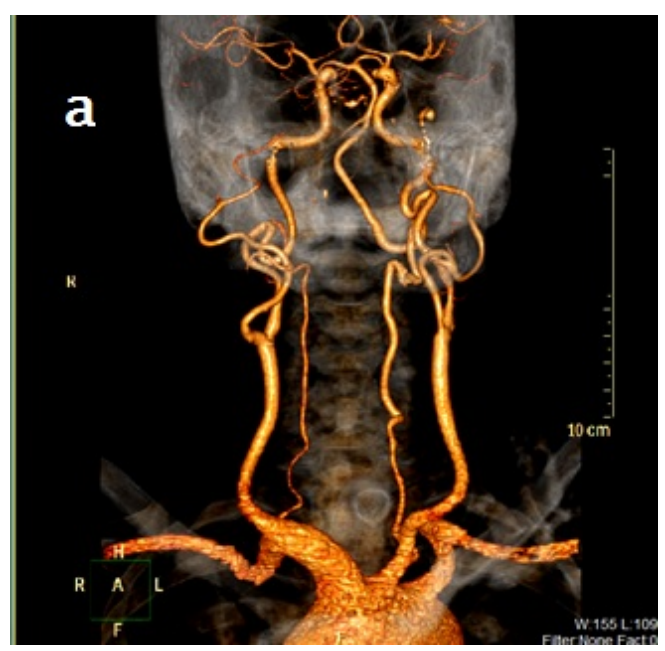

Figure 1: (a). Reconstructed 3D coronal CT head and neck angiography demonstrating diffuse atherosclerotic disease, with severe right vertebral disease, particularly at the V4 segment. 
Page 2 of 3

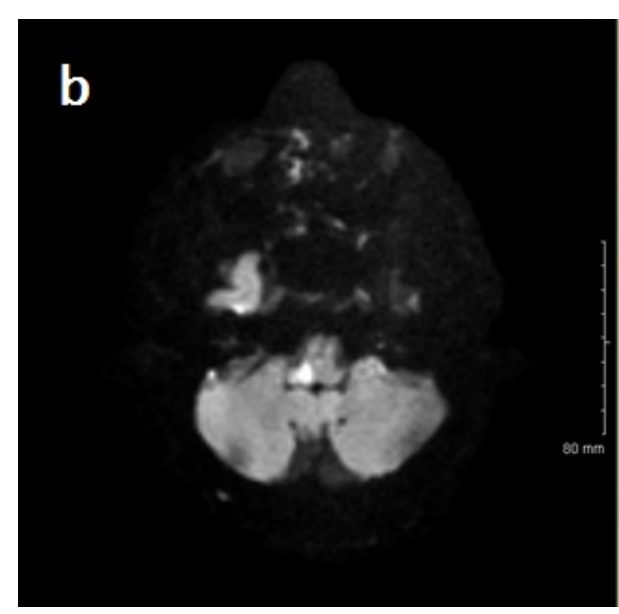

(b). Magnetic resonance imaging (Diffusion restriction imaging). Noted hyperintense area in the right lateral medulla, consistent with acute cerebral infarction in our patient.

However, very few cases of bilateral vocal cord dysfunction related to stroke have been described [11-14], and only two were in the setting of unilateral Wallenberg's syndrome (LMS), leading to upper airway obstruction $[9,12,13,15-17]$.

One of the largest cohort studies examining the incidence of vocal cord paresis in the setting of acute stroke reported VCP in 11 of the 54 patients (20.4\%) enrolled. Among these, there was a reported $100 \%$ incidence $(\mathrm{N}=5)$ of unilateral vocal cord paresis in lateral medullary infarction [14]. Of these affected patients, $80 \%(\mathrm{~N}=9)$ had ipsilateral VCP to their stroke [14]. However, none had bilateral vocal cord dysfunction.

Dysphonia was found in $16.7 \%$ of the patients and correlated strongly with the finding of VCP; and that all patients with dysphonia had VCP. However, out of the 11 patients with VCP; only 2 did not have dysphonia [14] [Table. 1].

\begin{tabular}{|l|l|}
\hline \multicolumn{1}{|c|}{ Dysfunction } & \multicolumn{1}{|c|}{ Effect } \\
\hline Vestibular nuclei & Vomiting, vertigo, nystagmus \\
\hline Inferior cerebellar peduncle & $\begin{array}{l}\text { Ipsilateral cerebellar dysfunction: ataxia, } \\
\text { dysmetria }\end{array}$ \\
\hline Ventral tegmental tract & $\begin{array}{l}\text { Palatal myoclonus, cardiac and pulmonary } \\
\text { dysautonomia }\end{array}$ \\
\hline Lateral spinothalamic tract & Contralateral deficit to pain, temperature \\
\hline Spinal trigeminal nucleus/tract & $\begin{array}{l}\text { Ipsilateral loss of pain, and temperature in V1- } \\
\text { V3 distribution }\end{array}$ \\
\hline Nucleus ambiguous & $\begin{array}{l}\text { Cardiac and pulmonary dysautonomias, } \\
\text { ipsilateral laryngeal, pharyngeal and palatal } \\
\text { dysfunction }\end{array}$ \\
\hline $\begin{array}{l}\text { Ascending parasympathetic } \\
\text { fibers }\end{array}$ & \begin{tabular}{l} 
Ipsilateral Horner's syndrome \\
\hline
\end{tabular} \\
\hline
\end{tabular}

Table 1: Summarizing findings in lateral medullary syndrome
We report a case of unilateral lateral medullary syndrome resulting in bilateral vocal cord dysfunction, leading to life-threatening upper airway obstruction. This case further highlights bilateral vocal cord paralysis as an unusual complication of lateral medullary syndrome; in which only two prior case reports have been described.

\section{Case}

A 75 year-old right handed, white Caucasian man, with a history of hypertension, without prior cardiac or neurological disease, presented to our institution because of a near-syncopal episode, associated with severe bradycardia, simultaneous acute dysarthria and dysphonia.

On admission, he was mildly hypertensive (BP 150/80) with $>95 \%$ oxygen saturation on room air. He was experiencing paroxysmal episodes of severe sinus bradycardia, as low as $20 \mathrm{bpm}$, intermittently requiring atropine.

Neurological examination revealed intact cortical function, sustained horizontal nystagmus on extreme right gaze, strained vocalization with severe hypophonia and dysarthria; along with loss of gag reflexes. He exhibited intact strength, with truncal ataxia and left sided dysmetria.

Magnetic resonance imaging of the head revealed acute infarction of the left lateral medulla as well as a punctate infarct in the right cerebellar hemisphere. Bilateral vertebral arterial stenosis was noted on CT angiography of the head and neck, with more prominent atherosclerotic burden in the left vertebral artery, particularly in the V4 segment, associated with an apparent intraluminal thrombus. Transthoracic echocardiography revealed normal left ventricular cavity size, preserved ejection fraction, mildly dilated left atrium, and mild aortic valvular regurgitation.

Due to the patient's delayed arrival, intravenous (IV) thrombolytic therapy was not administered. Instead, he was treated with rectal aspirin $325 \mathrm{mg}$, and was started on full dose parenteral anticoagulation with IV heparin (because of the perceived intraluminal thrombus).

The patient's condition worsened over the next 24 hours with progressive upper airway symptoms of worsening dysarthria, profound inability to clear secretions, as well as witnessed aspiration. He was intubated with fibre optic-assisted laryngoscopy for airway protection, with no documented trauma to the vocal cords during intubation. Repeat MRI 24 hours after admission showed stability of the prior findings without change.

ENT consultation, which was obtained for the evaluation of severe dysarthria, included flexible laryngoscopy that yielded limited views of the piriform sinuses in the hypopharynx secondary to copious mucopurulent secretions. Advancing the laryngoscope into the larynx revealed severe hypomobility of the true vocal folds bilaterally. Subsequently, this patient underwent tracheostomy placement for airway protection as well as percutaneous gastrostomy tube for nutritional support.

His hospital course was complicated by sepsis related to recurrent aspiration pneumonia, in addition to gastrostomy site cellulitis requiring repeated surgical re-exploration. The patient and his family eventually opted for hospice care.

\section{Discussion}

This case illustrates an important point: Bilateral vocal cord dysfunction is very rare in stroke, but may occur. Bilateral VCP, 
especially when not promptly addressed, can become life threatening, secondary to upper airway obstruction. Even with medical and surgical management, the rate of complications in patients with bilateral vocal cord paresis is high, especially pulmonary complications. A high index of suspicion of vocal cord dysfunction is advised when patients with stroke present with wheezing and dysphonia the latter being a reliable sign of vocal cord dysfunction, and an important clinical feature in the aspiration mechanism $[11,12,18,19]$.

As noted, bilateral vocal cord paralysis is most often iatrogenic, but may also be associated with inflammatory conditions and malignancy. Unilateral cortical, or subcortical stroke as a cause of bilateral VCP has also been described, but is extremely rare $[12,13]$. However, only two prior case reports of bilateral vocal cord paresis in the setting of Wallenberg's syndrome have been published in the literature [16,17].

Lower motor neuron innervation of the vocal cords originates from the nucleus ambiguous, situated along the reticular formation in the lateral medulla. Special visceral efferent fibers from the nucleus ambiguous exit the brainstem ipsilaterally between the inferior cerebellar peduncle and the olive. These then combine with other fibers to form the vagus nerve. The supranuclear innervation of the vocal cord and nucleus ambiguous are much less understood. It is theorized that there are both pyramidal and extrapyramidal contributions, with a large amount of redundancy and a disproportionately large amount of subcortical innervation to facilitate reflex laryngeal function, reflecting its importance $[14,20]$. Thus, the mechanism of bilateral vocal cord paresis in the setting of lateral medullary syndrome, with unilateral nucleus ambiguous involvement, remains unclear.

It has been reported that, in other animals (i.e., squirrels, monkeys), the corticobulbar laryngeal control pathway divides into two parts after synapsing in the ipsilateral dorsal reticular nucleus. One part projects directly into the ipsilateral nucleus ambiguous, and the other part crosses to the contralateral nucleus. However, the existence of such pathways in humans has not been documented.

In summary, this report highlights bilateral vocal cord paralysis as an unusual complication of lateral medullary syndrome. The exact underlying mechanism of this phenomenon remains unclear. Furthermore, neurologists and other physicians caring for patients with possible bilateral vocal cord paresis should be aware of the signs and symptoms that herald this condition, as even with optimal medical and surgical management, the morbidity and mortality associated with this condition remains high.

\section{References}

1. Marcet A (1811) History of a singular nervous or paralytic affection attended with anomalous morbid sensations. Medico-Chirurgical Transactions 2: 215-233.
2. Wallenberg A (1895) Akute Bulbäraffektion (Embolie der Arteria cerebelli post inf sinistra). Archives fur Psychiatry 27: 504-540.

3. Searls DE, Pazdera L, Korbel E, Vysata O, Caplan LR (2012) Symptoms and signs of posterior circulation ischemia in the new England medical center posterior circulation registry. Arch Neurol 69: 346-351.

4. Bulbaraffection (1901) (Embolie der Art. Cerebellar. Post. Inf. Sinister.)?" beschriebenen Falle. Arch Psychiat Nervenkr 34: 923-59.

5. Sörös P, Hachinski V (2012) Cardiovascular and neurological causes of sudden death after ischaemic stroke. Lancet Neurol 11: 179-188.

6. Kallmünzer B, Breuer L, Kahl N, Bobinger T, Raaz-Schrauder D, et al. (2012) Serious cardiac arrhythmias after stroke: incidence, time course, and predictors--a systematic, prospective analysis. Stroke 43: 2892-2897.

7. Jaster JH, Smith TW (1998) Arrhythmia mechanism of unexpected sudden death following lateral. medullary infarction. Tenn Med 91: 284.

8. Koay S, Dewan B (2013) An unexpected Holter monitor result: multiple sinus arrests in a patient with lateral medullary syndrome. BMJ Case Rep 2013.

9. Rosenthal LH, Benninger MS, Deeb RH (2007) Vocal fold immobility: a longitudinal analysis of etiology over 20 years. Laryngoscope 117: 18641870 .

10. Benninger MS, Gillen JB, Altman JS (1998) Changing etiology of vocal fold immobility. Laryngoscope 108: 1346-1350.

11. Caplan L (2000) Posterior circulation ischemia: then, now, and tomorrow. The Thomas Willis Lecture-2000. Stroke 31: 2011-2023.

12. Shaw GL (1987) Airway obstruction due to bilateral vocal cord paralysis as a complication of stroke. South Med J 80:1432-1433.

13. Ito Y, Mori A, Yonemura K, Hashimoto Y, Hirano T, et al. (2008) Upper airway obstruction with bilateral vocal cord paralysis secondary to ischemic stroke: report of two cases. Rinsho Shinkeigaku 48: 333-337.

14. Venketasubramanian N1, Seshadri R, Chee N (1999) Vocal cord paresis in acute ischemic stroke. Cerebrovasc Dis 9: 157-162.

15. Merati AL, Halum SL, Smith TL (2006) Diagnostic testing for vocal fold paralysis: survey of practice and evidence-based medicine review. Laryngoscope 116: 1539-1552.

16. Chung SJ, Yi H, Song TJ, Song D (2012) A Case of airway obstruction caused by bilateral vocal cord palsy in a patient with lateral medullary syndrome. Korean J Stroke 14: 89-91.

17. Mishra A, Dave N, Mehta M (2013) Lateral medullary syndrome presenting with acute central stridor secondary to bilateral vocal cord palsy. Int J Nutr Pharmacol Neurol Dis 3: 318-9.

18. Daniels SK, Brailey K, Priestly DH, Herrington LR, Weisberg LA, et al. (1998) Aspiration in patients with acute stroke. Arch Phys Med Rehabil 79: 14-19.

19. Horner J, Massey EW, Riski JE, Lathrop DL, Chase KN (1988) Aspiration following stroke: Clinical correlates and outcome. Neurology 38: 1359-1362.

20. Aronson AE (1990) Clinical Voice disoders. (3stedn), Thieme, New York 71-115. 